\title{
Médiévales
}

Langues, Textes, Histoire

75 | automne 2018

Traductions du Moyen Âge

\section{Sur une traduction médiévale de la Lettre aux frères du Mont-Dieu. Le témoignage ancien d'un vernaculaire savant}

A medieval translation of William of Saint-Thierry's Letter to the Brothers of Mont-Dieu. Early evidence of a learned vernacular

\section{Marie-Pascale Halary}

\section{OpenEdition \\ Journals}

Édition électronique

URL : https://journals.openedition.org/medievales/9349

DOI : $10.4000 /$ medievales. 9349

ISSN : 1777-5892

\section{Éditeur}

Presses universitaires de Vincennes

\section{Édition imprimée}

Date de publication : 15 octobre 2018

Pagination : 137-150

ISBN : 978-2-84292-861-2

ISSN : 0751-2708

\section{Référence électronique}

Marie-Pascale Halary, "Sur une traduction médiévale de la Lettre aux frères du Mont-Dieu. Le

témoignage ancien d'un vernaculaire savant », Médiévales [En ligne], 75 I automne 2018, mis en ligne le 15 octobre 2019, consulté le 22 avril 2022. URL : http://journals.openedition.org/medievales/9349 ;

DOI : https://doi.org/10.4000/medievales.9349 
Marie-Pascale Halary

\title{
Sur une traduction médiévale de la Lettre aux frères du Mont-Dieu Le témoignage ancien d'un vernaculaire savant
}

\author{
Dans une étude qui a fait date et qui est consacrée aux rapports \\ entre le latin et le français ainsi qu'à la place du vernaculaire dans la \\ pensée linguistique médiévale, Serge Lusignan indiquait ${ }^{1}$ :
}

Certains registres d'expression sont fermés au français. Le latin s'identifie entre autres au savoir et par conséquent à l'écrit. [...] Le XIII ${ }^{\mathrm{e}}$ siècle ne met pas en doute que le latin soit la langue unique de la philosophie, de la théologie et aussi de la liturgie (Jean le Danois, S. grammatica, p. 3). Tous ces domaines échappent au français comme à la limite ils échappent aux laïcs. Cela conduit Henri de Crissey à poser en opposition parallèle latin et vernaculaire, clerc et laïc : deux langues, deux classes sociales, deux fonctions. Dans cette perspective, le rapport du latin au français est asymétrique et on devrait parler à ce propos de diglossie plutôt que de bilinguisme.

L'analyse selon laquelle la langue latine et la langue romane, respectivement définies comme la langue haute et la langue basse, entretiennent une relation de distribution fonctionnelle a depuis été nuancée. Dans un ouvrage plus récent qui ressortit à une approche sociolinguistique ${ }^{2}$, l'historien, s'appuyant sur les travaux de Michel Banniard ${ }^{3}$, insiste sur le

1. S. Lusignan, Parler vulgairement. Les intellectuels et la langue française aux XIII et XIV siècles, Paris/Montréal, 1986, p. 83.

2. ID., Essai d'histoire sociolinguistique. Le français picard au Moyen Âge, Paris, 2012, p. 32-39.

3. Pour une critique de la notion de diglossie appliquée au Moyen Âge, voir en particulier M. BANNIARD, Viva voce. Communication écrite et communication orale du Iv au IXe siècle en Occident latin, Paris, 1992, p. 508-511 ; ID., " Du latin des illettrés au roman des lettrés. La question des niveaux de langue en France (VIII-XII ${ }^{\mathrm{e}}$ siècle) », dans P. von Moos éd., Zwischen Babel und Pfingsten/Entre Babel et Pentecôte. Schprachdifferenzen und Gesprächverständigung in der Vormoderne (8.-16. Jahrhundert)/Différences linguistiques et communication orale avant la modernité (VIII $-\mathrm{XVI}^{e}$ siècle), Zurich/Berlin, 2008, p. 269-286. Voir aussi B. GRÉvin, Le Parchemin des cieux. Essai sur le Moyen Âge du langage, Paris, 2012. 
statut qu'il faut accorder aux discours médiévaux proclamant la supériorité du latin, voire l'incapacité du vernaculaire à assumer certains registres savants : à l'instar des propos de Jean le Danois ou de Henri de Crissey, il s'agit de représentations cléricales qui, si elles ne sont évidemment pas infondées, ne permettent peut-être pas de décrire toutes les pratiques linguistiques.

Pour contester la pertinence du modèle diglossique, il est effectivement possible de revenir sur l'origine des discours sur les langues au Moyen Âge. Mais on peut aussi examiner les textes eux-mêmes : certains d'entre eux, même de manière très marginale, montrent que le latin n'est pas l'idiome exclusif de la haute spéculation philosophique et religieuse - domaines dont on pourrait $a$ priori penser qu'ils sont parmi les derniers des différents registres de l'écriture à être concernés par l'extension progressive de la langue française.

C'est à l'un de ces textes, la translation ancienne ( $\mathrm{XII}^{\mathrm{e}}$ siècle) d'un traité de Guillaume de Saint-Thierry, que cette étude voudrait s'intéresser. À partir de quelques exemples précis, je voudrais revenir sur la manière dont le traducteur résout certaines difficultés liées à la mise en roman d'un discours savant.

\section{La langue de la traduction, une haute langue vernaculaire}

Il convient de commencer par rappeler certains éléments de contexte concernant les translations monastiques du $\mathrm{XII}^{\mathrm{e}}$ siècle. On a depuis longtemps repéré sur le territoire de la France continentale ${ }^{4}$ différentes traductions monastiques conservées pour la plupart dans des manuscrits uniques et anciens ( $\mathrm{XII}^{\mathrm{e}} \mathrm{Ou} \mathrm{XIII}^{\mathrm{e}}$ siècle) dont l'existence même n'a rien d'une évidence. Il s'agit de la Lettre aux frères du Mont-Dieu de Guillaume de Saint-Thierry ${ }^{5}$, de plusieurs textes de Grégoire le Grand ${ }^{6}$ ou de Bernard de Clairvaux (parmi lesquels on compte une partie des Sermons sur le Cantique des cantiques et le De diligendo Deo, textes qu'on imagine plutôt destinés à un public monastique $)^{7}$, du manuscrit bilingue qui présente les

4. Le cas de l'anglo-normand est spécifique en raison de la situation linguistique de cette région : voir surtout I. SHORT, « Patrons and Polyglots: French Literature in TwelfthCentury England », Anglo-Norman Studies, 16 (1992), p. 229-249.

5. Li Espistle saint Bernart a Mont Dev, dans Die Epistola ad fratres de Monte Dei des Wilhem von Saint-Thierry, Lateinische Überlieferung und mitterlaterliche Übersetzungen, éd. V. Honemann, Zürich/Munich, 1978.

6. Li Dialoge Gregoire lo pape et Moralités sur Job, éd. W. Foerster, Halle/Paris, 1876.

7. Predigten des h. Bernhard in altfranzösischer Übertragung, aus einer Handschrift der königlichen Bibliothek zu Berlin, éd. A. Schulze, Tübingen, 1894 ; Li Sermon Saint Bernart. Älteste französische Übersetzung der lateinischen Predigten Bernhards von Clairvaux nach der Feuillantiner Handschrift in Paris, éd. W. Foerster, Erlangen, 1885 ; La Traduction en prose française du XII siècle des Sermones in Cantica de saint Bernard, éd. G. Stewart, Amsterdam, 1994. Les autres traductions ne sont pas éditées. 
versions latine et française des Synonyma d'Isidore de Séville ${ }^{8}$ et, sans doute, d'une traduction du Benjamin minor de Richard de Saint-Victor' ${ }^{9}$. Toutes ces translations ont été rapprochées dans plusieurs études car on y a reconnu un " groupe [...] homogène ${ }^{10}$ » qui rassemble des « traductions monastiques $^{11}$ » composées vers 1150-1200 dans l'est et le nord-est de la zone d'oïl ${ }^{12}$. Ces mises en roman sont étonnantes à plus d'un titre. Non seulement elles comptent parmi les premières productions en prose française, mais aussi elles translatent de manière littérale et en recourant à un lexique en partie commun ${ }^{13}$ des textes spirituels dont plusieurs figurent parmi les grands traités de la littérature monastique du XII ${ }^{\mathrm{e}}$ siècle.

De ce fait, ce petit corpus, qui porte témoignage d'une activité traductrice aux confins de la Wallonie et de la Lorraine pendant la seconde moitié $\mathrm{du} \mathrm{XII}^{\mathrm{e}}$ siècle, permet de mettre en lumière, bien avant le grand mouvement des traductions savantes, une autre conjoncture historique qui a sans doute favorisé l'émergence d'une haute langue vernaculaire. Étant donné que le choix de la langue romane n'est guère réductible ici à une simple volonté d'édification du « vulgaire », l'existence de ce corpus invite à ne pas associer systématiquement vernacularisation et vulgarisation (ces translations n'adaptent pas le contenu spéculatif des traités latins) et à revenir sur la notion de diglossie. Plus encore, ce petit groupe de manuscrits attire l'attention sur le rôle que purent avoir les traductions dans la naissance et le développement de la prose française et, plus largement, sur l'importance des échanges et des transferts linguistiques pour modéliser le changement et penser l'irruption d'une nouveauté telle que celle de la prose romane ${ }^{14}$.

8. F. Bonnardot, « Dialogus Anime Conquerentis et Rationis Consolantis, traduction en dialecte lorrain du XII ${ }^{\mathrm{e}}$ siècle », Romania, 5 (1876), p. 269-332. La traduction en prose romane est inachevée.

9. G. HASENOHR, « Sur une ancienne traduction lorraine (XII ${ }^{\mathrm{e}} \mathrm{s}$.) du Benjamin minor » [1991], dans EAD., Textes de dévotion et lectures spirituelles en langue romane (France, XII XVI siècles), Turnhout, 2015, p. 479-485.

10. M. Carieri, C. Ruby, I. Short, Livres et écritures en français et en occitan au XII siècle, Rome, 2011, p. XLV.

11. G. Hasenohr, « Sur une ancienne traduction lorraine... », p. 483.

12. Sur ce groupe, outre les références déjà indiquées, voir l'introduction de B. WOLEDGE et H. P. Clive, Répertoire des plus anciens textes en prose française depuis 842 jusqu'aux premières années du XIIt siècle, Genève, 1964, p. 9-44 ; A. HENRY, « Saint Bernard traduit vers 1200 en pays wallon », Les Dialectes de Wallonie, 8-9 (1981), p. 95-111 ; G. PARADISI, La parola e l'amore. Studi sul Cantico dei Cantici nella tradizione francese medievale, Rome, 2009, p. 51-70.

13. Voir notamment G. HASENOHR, « Sur une ancienne traduction lorraine... ».

14. Sur ce point, voir la théorie dite du «polysystème » telle qu'elle a été développée par I. Even-Zohar (« Polysystem Theory (revised) », dans ID., Papers in Culture Research [2005], livre électronique, 2010, p. 40-50 ; ID., « The Position of Translated Literature within the Literary Polysystem », Poetics today, 11.1 (1990), p. 45-51 (version révisée de l'article publié dans Literature and Translation. New Perspectives in Literary Studies, Louvain, 1978, p. 117-127). Cette théorie a souvent été sollicitée pour analyser les littératures médiévales du Nord de l'Europe ; elle est moins exploitée pour la production vernaculaire d'oïl. 
Toutefois, si ces textes montrent qu'à une date ancienne, dans la zone continentale d'oïl, il a existé - même de manière très ponctuelle un haut discours spirituel en langue vernaculaire, nombreuses sont les investigations qui restent à mener. Qui fut exactement le public de ces manuscrits, susceptibles d'interroger l'identification que l'on fait le plus souvent entre la langue romane et les laïcs ? De même, comment expliquer l'existence d'un tel corpus et quels furent les réseaux de diffusion de ces traductions, rassemblées sur une zone géographique précise ? S'il n'est pas possible, dans le cadre de cette étude, de revenir sur ces points, cette contribution voudrait, à partir d'un exemple, préciser certaines des modalités de traduction qui sont celles de ces textes.

\section{Li Epistle saint Bernart a Mont Deu}

La translation de la Lettre aux frères du Mont-Dieu est une des plus anciennes versions françaises d'un traité monastique. La version latine, attribuée au liégeois Guillaume de Saint-Thierry, fut sans doute rédigée en 1144 pendant un séjour à Signy ${ }^{15}$. Ce texte, qui consiste en une réflexion sur l'idéal monastique et cartusien, prend la forme d'une lettre, adressée aux frères de la Chartreuse du Mont-Dieu, dont la deuxième partie fait la part belle à « la théorie », avec « toute une anthropologie et toute une théologie parfois subtile et compliquée ${ }^{16} »$. L'étude, par Jean Déchanet, de la diffusion de ce traité ${ }^{17}$ a montré que celle-ci, très large, avait été en grande partie assurée par les cisterciens et que le nom de Bernard s'était souvent substitué à celui de Guillaume. Quant aux versions vernaculaires, si on connaît des adaptations germaniques plus tardives ${ }^{18}$, la plus ancienne de toutes est la version d'oïl conservée par le manuscrit de Verdun (BM, 72), daté de la deuxième moitié du XII ${ }^{\mathrm{e}}$ siècle $^{19}$. S'appuyant sur sa scripta, qu'il identifie comme lorraine, Volker Honemann fait l'hypothèse que cette version de « li epistle saint Bernart a Mont $\mathrm{Deu}^{20}$ » a peut-être été copiée à Saint-Paul de Verdun ${ }^{21}$.

15. J. Déchanet, «Introduction » à son édition de Guillaume De SAint-Thierry, Lettre aux frères du Mont-Dieu (Lettre d'or) [1975], Paris, 2004, p. 26 ; P. VERDEYEN, Guillaume de Saint-Thierry, premier auteur mystique des anciens Pays-Bas, trad. A. Louf, Turnhout, 2003.

16. J. DéCHANET, «Introduction »..., p. 36.

17. Ibid., p. $50 \mathrm{sq}$.

18. Voir V. Honemann, Die Epistola...

19. Outre l'étude de V. HonemanN, voir la description consacrée à cette traduction dans M. CARIERI, C. Ruby, I. Short, Livres et écritures..., p. 222-223, et la notice rédigée par P.M. JoRIS dans C. GALDERISI éd., Translations médiévales. Cinq siècles de traductions en français au Moyen Âge (XI -XVe siècles) : étude et répertoire, Turnhout, 2011, vol. 2, t. I, p. 528-529.

20. Je cite d'après l'édition diplomatique faite par V. Honemann. La ponctuation et la distinction $u / v$ et $i / j$ sont miennes.

21. Ibid., p. 102-111. 
Une étude rapide montre que, comme les autres traductions monastiques, la translation de la Lettre d'or est extrêmement proche du texte latin et qu'elle procède mot à mot. Sans une comparaison précise qui prenne en compte toutes les données de la tradition manuscrite, il est même difficile de conclure à des différences entre le latin et la langue dite vulgaire, les omissions ou les déplacements repérés à partir de l'édition latine établie par Jean Déchanet pouvant s'expliquer par l'identité du manuscrit source ${ }^{22}$. Partant de cette proximité entre les deux langues, il est possible d'examiner quelques-unes des solutions adoptées par la version romane quand il s'agit de traduire un latin savant.

Si les jeux verbaux en latin sont moins nombreux chez Guillaume de Saint-Thierry que chez Bernard de Clairvaux ${ }^{23}$, il n'en demeure pas moins que le texte source propose plusieurs développements qui reposent sur la reprise d'un paradigme morphologique et/ou sur des échos phoniques. Il en est ainsi, par exemple, de la formule nourrie de la réminiscence de $J n 3,8^{24}$, répétée dans le $\S 251$ : «Spiritus sanctus, qui ubi vult spirat, quando vult et quomodo vult, et quibus vult, in hoc spirat », « [...] cum audierit vocem spiritus spirantis » (Epistola..., p. 344). Utilisant les ressources du français, le translateur ne manque pas, le plus souvent, de rendre de tels jeux : «c'est quant li sainz esperiz ki espiret ou k'il vuet anluminet an ceu ceos k'il vuet, et quant il vuet, et ansi cum il vuet », « apermeimes ke li cuers averat oit la voix de l'esperit espirant» (Li Epistle..., p. 273). De même, le travail autour des dérivés latins de mutare est rendu par de semblables constructions, sur mиеr:

[Deus] mutabilia omnia immutabiliter faciens. (Epistola ..., p. 380)

[Deus] faisant totes muaules choses sanz muauleteit. (Li Epistle ..., p. 280)

Ces deux petits exemples le montrent ${ }^{25}$ : la traduction du traité est précise, littérale et elle témoigne d'une bonne compréhension du texte latin.

22. Les $§ 7-14$ du billet d'envoi de l'édition J. Déchanet ne sont pas translatés en français (ce qui est sans doute à mettre en relation avec les deux rédactions successives et, partant, les deux versions de la préface). On note également des différences, par exemple, pour les $§ 161$, 190,207 , etc. Les deux versions sont toutefois suffisamment proches et la traduction française est suffisamment littérale pour qu'une comparaison entre l'édition de J. Déchanet et le texte du ms. de Verdun soit probante.

23. Voir par exemple J. LECLERCQ, Recueil d'études sur saint Bernard et ses écrits, Rome, 1969, t. III, p. 145-162.

24. «Spiritus ubi vult spirat, et vocem ejus audis, sed nescis unde veniat, aut quo vadat: sic est omnis qui natus est ex spiritu. »

25. Voir aussi le $\S 31$ (Epistola...,p. 168) : les moines habitent-ils dans les cieux ou dans des cellules (« in caelis », « in cellis », deux termes qui viennent de celare) ? Le français reproduit le jeu : «Por ceu si habiteiz vos anceos en ciel c'an celles selonc la forme de vostre proposemant. [...] Et ciel et celle dist an et por ceu les apelet om ensi k'il recelent car ceu ke receleit est an ciel est ausi receleit an celles » (Li Epistle..., p. 230). 


\section{La traduction du binôme anima/animus dans Li Epistle}

Dans ce contexte, il est un passage dont il est intéressant d'étudier la translation car à la difficulté de traduire un vocabulaire latin savant s'ajoute un deuxième problème, lié à la proximité entre les deux substantifs latins anima et animus. Dans le développement sur l'homme rationnel, Guillaume commence par annoncer que cet état correspond à celui où l'animus est raisonnable ( « rationalis »). Voulant apporter des précisions sur « cette âme que la raison rend raisonnable ${ }^{26} »$, il indique qu'il expliquera d'abord ce qu'est l'anima. Suit alors le $\S 198$ qui, me semble-t-il, constitue un véritable défi pour la prose vernaculaire :

Anima est res incorporea, rationis capax, vivificando corpori accomodata. Haec animales constituit homines, quae carnis sunt sapientes, sensibus corporis inhaerentes. Quae ubi perfectae rationis incipit esse, non tantum capax, sed et particeps, continuo abdicat a se notam generis feminini, et efficitur animus particeps rationis, regendo corpori accomodatus, vel seipsum habens spiritus. Quamdiu enim anima est, cito in id quod carnale est effeminatur ; animus vero, vel spiritus, non nisi quod virile est et spirituale meditatur. (Epistola..., p. 306-308) ${ }^{27}$

Selon une première analyse et comme pour l'ensemble du traité, le problème peut sembler être avant tout conceptuel et lexical. Le français peut-il rendre un adjectif comme « incorporeus » ou une formule telle que « rationis capax » et quelles sont les possibilités qui se présentent ? Dans ce passage, cette difficulté est comme redoublée par un obstacle linguistique : Guillaume s'appuie sur la distinction latine entre le substantif féminin anima et le substantif masculin animus ${ }^{28}$ pour établir, conformément à la hiérarchie des genres présente dans les représentations médiévales ${ }^{29}$, une progression de l'anima à l'animus. Le perfectionnement de l'anima, ce serait l'animus. En effet, la première, seulement « capable de raison », est associée à ce

26. « [...] animus ipse, quem ratio rationalem facit » (trad. J. DÉCHANET, Epistola ..., p. 306).

27. "C'est une substance incorporelle, capable de raison, propre à donner la vie au corps. L'âme animale rend "animaux" ceux des hommes qui s'affectionnent aux choses de la chair et s'assujettissent aux sens du corps. Quand cette âme commence à être non seulement capable, mais aussi doué d'une raison parfaite, elle rejette aussitôt loin d'elle l'indice du genre féminin : d'anima elle devient animus, âme raisonnable, propre à diriger le corps ; esprit qui se possède lui-même. Aussi longtemps qu'elle reste anima, elle s'effémine volontiers en se portant vers le charnel ; une fois animus au contraire, une fois esprit, elle ne s'arrête plus qu'aux choses viriles et spirituelles » (ibid., p. 307-309).

28. Sur cette distinction, voir M.-M. Davy, dans Guillaume De Saint-Thierry, Un traité de la vie solitaire, lettre aux frères du Mont-Dieu [1940], Paris, 1946, p. 147-149.

29. Sur l'infériorité de la femme, voir par exemple D. LetT, Hommes et femmes au Moyen Âge. Histoire du genre (XII -XVe siècle), Paris, 2013. 
qui est animal et charnel : ce paradigme est indexé au féminin ${ }^{30}$. L'animus, quant à lui, correspond à un état plus spirituel, dans lequel cette « capacité » de raison est comme actualisée (《particeps rationis ») $)^{31}$.

Or un tel passage, qui associe à la dimension spéculative une différence anima/animus que le français ne possède pas $^{32}$, ne manque pas d'être intégralement traduit dans la langue dite vulgaire par le translateur du XII ${ }^{\mathrm{e}}$ siècle :

Airme est une chose niant corporeis, covenaule por receoure raison, doneie au cors por lui a vivifier. Ceste fait les homes animaus, k'il nen assavorent se chair nun et ki a sanz de lor cors sunt aihers. Mais quant ceste airme encomancet ai estre parceniere de raison, apermeimes gittet ansus de lei lo feminien nom et se devient cuers parceniers de raison, doneiz a cors por regneir sor lui, ou ale devient esperiz posseans lui meimes. Tant cum li airme est airme, se decourt ale tost an ceu ke cherneis chose est. Mais quant ale est devenue cuers ou esperiz, se ne panset ele se bernil chose nun et esperiteil. (Li Epistle..., p. 264)

Cette version vernaculaire témoigne à la fois d'une bonne compréhension du latin et d'une préférence pour les équivalents français (plutôt que pour les emprunts) : c'est en ce sens que peut être comprise la présence de covenaule (pour « capax »), de aihers, « attaché » (pour « inhaerentes ») ou de parcenier, « celui qui participe, qui prend part» (pour «particeps ») ${ }^{33}$. Les exemples donnés par les dictionnaires montrent que ces termes sont bien attestés au XII ${ }^{\mathrm{e}}$ siècle ${ }^{34}$. De même, la formule « vel seipsum habens spiritus » est rendue par « ou ale devient esperiz posseans lui meimes » et le parallèle « vivificando corpori accomodata »/« regendo corpori accomodatus » est perçu et restitué : «doneie au cors por lui a vivifier »/《doneiz a cors por regneir sor lui ». La translation emploie parfois des équivalents ou des constructions un peu différentes mais elle révèle une saisie souvent très juste du contenu du propos autant qu'une tendance à recourir aux ressources propres de la langue vernaculaire. Comme cela a

30. Sur ce paradigme, voir P. Buc, L'Ambiguïté du Livre. Prince, pouvoir, et peuple dans les commentaires de la Bible au Moyen Âge, Paris, 1994, p. 71 sq.

31. Cette définition de l'animus doit beaucoup à Augustin qui, après avoir rappelé le caractère incorporel de celui-ci, indique : «Nam mihi videtur esse substantia quaedam rationis particeps, regendo corpori accommodata » (De quantitate animae, dans Euvres de saint Augustin, V. Dialogues philosophiques, texte de l'édition bénédictine, Paris, 1948, p. 272).

32. De manière significative, la traduction moderne citée en $n .27$, qui ne peut trouver des équivalents français, reprend les termes latins.

33. Parcenier est un mot construit à partir de partitio : voir W. von Wartburg éd., Französisches etymologisches Wörterbuch. Eine Darstellung des galloromanischen Sprachschatzes, Tübingen, 1955, Band 7, p. 691-692.

34. A. Tobler et E. Lommatzsch, Alffranzösisches Wörterbuch, Wiesbaden, 1925-, t. I, col. 163-165, t. II, col. 976, et t. VII, col. 210-212 ; F. Godefroy, Dictionnaire de l'ancienne langue française du IX au XV siècle, Genève/Paris, 1982 (reprod. de l'éd. Paris, 1891-1902), t. IX, p. 189 , t. V, p. 749-751. 
déjà été signalé ${ }^{35}$, rien ne le montre mieux que la manière dont le traducteur rend les mots négatifs du type incorporeus. S'il lui arrive, parfois, de calquer le système de préfixation latin ${ }^{36}$, il construit le plus souvent ces termes à l'aide des morphèmes négatifs niant (pour les adjectifs et les participes passés) et non (en général pour les substantifs ou les équivalents) ${ }^{37}$. De là, la traduction « chose niant corporeis » pour « res incorporea $»^{38}$. On le voit donc, ce qui se déploie dans un tel texte, c'est bien une langue savante vernaculaire - plus « romane », pourrait-on dire, que la langue savante qui lui succède et qui emprunte bien davantage au latin.

Dans ce contexte, quelle est la solution choisie pour rendre la distinction anima/animus ? En réalité, la version française conserve l'opposition féminin/ masculin (qui, on l'a vu, est pertinente puisque tout le raisonnement se fonde sur les paradigmes antithétiques et hiérarchiques femme-charnel versus homme-spirituel), mais elle perd le jeu morphologique entre anima et animus : dans le passage cité, le substantif latin féminin est translaté par le nom français, également féminin, airme ( âme ») tandis que le mot animus est traduit les deux fois par cuer. À l'échelle du traité, ce choix de traduction est d'ailleurs extrêmement cohérent : même si le terme peut être l'équivalent vernaculaire d'autres mots latins ${ }^{39}$, il faut noter la constance avec laquelle animus est rendu par cuer, aussi bien avant le passage qui pose la différence entre animus et anima $^{40}$ qu'après celui-ci ${ }^{41}$.

35. G. HASENOHR, « Retour sur les caractères linguistiques du manuscrit de Chantilly et de ses ancêtres », dans S. L. Field, R. E. Lerner, S. Piron éd., Marguerite Porete et le Miroir des simples âmes. Perspectives historiques, philosophiques et littéraires, Paris, 2013, p. $103-126$ (p. 112).

36. Voir ainsi inobediance (Li Epistle..., p. 247, 268) et inobedianz (ibid., p. 235, 271), inperfectiun (ibid., p. 276) et tout le paradigme de dessemblance (dissamblant, dissamblamment mais niant dissamblamment (ibid., p. 275).

37. Voici un relevé, le chiffre entre parenthèses indiquant le paragraphe, qui est le même pour les éditions latine et française :

indefessa : niant lasseie (§ 29); nondum illuminata : niant ancor anlumineie (§ 49); informem : niant formeie ( $(50)$; jugis : niant defaillanz $(\$ 181)$; non ficta : niant fointe ( $\$ 214,264)$; inutilitia : niant profitaules $(\$ 37,220)$; indisciplinatis : niant disciplineies (§ 247); incommutabilis : niant cheiniaule ( $\$ 276,277,283$, etc.); non supervolitans : niant sorvolanz ( $(277)$; illocalis : niant local ( $(294)$; mais indignos : nundignes $(\S 23,252$; $\S 118$ : substantivé) ; instabilem : nonstaule ( 120$)$; ignorat : ele soit nunsaicheanz ( $(143)$; inscios : nonsaicheant ( $\$ 165,185)$; infidelibus : nonfeol ( $\$ 160)$.

ignorantia : nonsachance $(\S 204)$; instabilitas : nonstauleteiz $(\$ 223)$; indiscretio : nondiscretions (\$ 229); non valendi : nunpooir.

38. On peut trouver l'emprunt incorporel au XII ${ }^{\mathrm{e}}$ siècle (le Complément de Godefroy le signale dans la Chronique des ducs de Normandie), mais les exemples donnés par les dictionnaires sont en général plus tardifs : Gautier de Coinci, Oresme, Corbechon, Ciboule. Voir surtout K. BALDINGER éd., Dictionnaire étymologique de l'ancien français, Québec/ Tübingen, 1971, t. I, col. 184.

39. Cuer traduit régulièrement cor, dont il provient $(\S 1,86,173,216)$, et mens $(\S 77,137$, 244).

40. Voir ainsi, pour les éditions latine et française, les § 44, 69, 87, 95, 96, 120, 172.

41. Voir, outre les passages qui seront ensuite évoqués, les $\S 206$ (pour le GN « li cuers », l'édition latine donne «mens vel animus », p. 312), 208, 214, 220. 


\section{La traduction du binôme anima/animus dans les " traductions monastiques"}

Faut-il s'étonner de cette distribution anima/animus en airme/cuer et ne l'expliquer que par cet impératif d'une discrimination de genre ? Avant tout, il convient d'essayer de repérer quelles sont les solutions qui s'offrent à la langue du XII ${ }^{\mathrm{e}}$ siècle. Des sondages au sein du groupe des traductions monastiques montrent une forte tendance à traduire respectivement anima et animus par airme et corage. Voici un extrait du texte d'Isidore de Séville, puis sa traduction, remarquable par ses échos lexicaux avec la version française de Guillaume ${ }^{42}$ :

Nullus est qui in hac vita positus non suspiret : «Malis omnia plena sunt ! » Interpone ergo tibi rationem, particeps esto rationi[s], prevaleat ratio. Tempera animum ratione, animam ratione confirma ; vim tanti meroris reprimat ratio ; confirmato animo nullum periculum pertimescas.

Nus n'est mis en ceste vie qui ne sospire : «Totes choses sunt plenes des mais ! » Entremas le [raison] a toy, et soies parcinirs, et venket toi raisons. Atempre to corage et confarme t'anime par raison, li quele rapresse la force de si grant plor : n'aies dote de nul peril par confarmé corage.

Comme auparavant, le latin particeps est translaté par « parcinirs », tandis que « corage » est l'équivalent vernaculaire d'animus, traduction qu'on retrouve dans l'ensemble de ce texte ${ }^{43}$ comme dans de nombreuses mises en roman contemporaines ${ }^{44}$. Plus encore, l'examen de glossaires latinfrançais, quoique plus tardifs, révèle la constance avec laquelle coraige est le mot vernaculaire correspondant à animus. C'est ce que l'on observe dans ces lexiques toutes les fois où existe une entrée « animus »: dans le manuscrit Città del Vaticano, BAV, lat. 2748 (début du XIV ${ }^{\mathrm{e}}$ siècle) pour l'Abavus ${ }^{45}$, et dans le manuscrit Paris, BnF, lat. 13032 (fin du XIV siècle) pour l'Aalma ${ }^{46}$.

42. Dialogus Anime Conquerentis et Rationis Consolantis, p. 284-285 (auquel j'emprunte également le latin). Je souligne.

43. Pour d'autres exemples, voir ibid., p. 276-277 et 278-279.

44. Voir ainsi « Deux fragments d'Homélies sur Ezéchiel », éd. J. Chaurand, Romania, 88 (1967), p.91-112 (p. 100 et 103); Li Dialoge Gregoire lo pape, p. 5, p. 7, etc.; La Traduction en prose française du XII siècle des Sermones in Cantica de saint Bernard, p. 41, p. 83, etc. ; la traduction du De diligendo Deo, Nantes, Musée Dobrée, ms. 5, f. 172c.

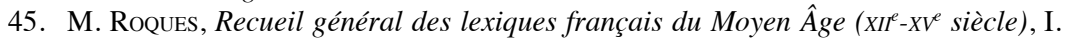
Lexiques alphabétiques [1936-1938], Paris, 1969-1970, t. I, p. 103. Les autres manuscrits de l'Abavus ne donnent pas ce lemme.

46. Ibid., t. II, p. 19. Le dépouillement des ouvrages de Firmin Le Ver et de Guillaume Le Talleur aboutit au même résultat, même si l'établissement des synonymies « animus vel audacia » et « hardiesse, courage » dans le premier révèle une évolution sémantique. 
Dans un tel contexte où, pour le vocabulaire vernaculaire savant, il semble bien établi qu'animus équivaut à corage, il s'agit de comprendre le choix du traducteur de Guillaume qui rend toujours le substantif latin par cuer. En réalité, il me semble préférable de voir dans les traductions par cuer et par corage deux variantes pour une même solution plutôt que deux translations véritablement différentes. La relation entre cuer et son dérivé correspond bien, en effet, à la situation décrite par Georges Merk parlant d'une « indifférenciation sémantique [...] manifeste lorsqu'un substantif suffixé est en concurrence avec le même substantif à suffixe zéro ${ }^{47}$ ». Autrement dit, l'emploi de l'une ou l'autre des deux formes, senties comme des quasi synonymes, serait indifférent du point de vue du sens et on pourrait émettre différentes hypothèses, qui ne sont pas incompatibles, pour expliquer la présence de cuer dans la traduction de la Lettre d'or. Tout d'abord, même si une interprétation exclusivement chronologique n'est pas suffisante (dans la mesure où la translation par corage est bien attestée dans les textes anciens), on peut faire la proposition d'une tendance qui conduirait corage à se substituer à cuer : outre que la courbe de productivité du suffixe -age est basse en ancien français, au moins un autre texte contemporain, la translation des Sermones per annum de Bernard de Clairvaux ${ }^{48}$, préfère cuer pour rendre le latin animus. Un autre élément, la valeur phonique du substantif, est peut-être à prendre en compte : dans le passage concerné de la Lettre qui, comme d'autres extraits du traité, porte aussi sur les rapports avec le corps, on ne peut pas exclure que s'exerce une pression qui favorise le substantif cuer, monosyllabe en [k], souvent associé au nom cors dans les textes vernaculaires ${ }^{49}$. Enfin, si corage est incontestablement un nom masculin, sa terminaison en - $e$ (qui, dans le système français, peut être un morphème du féminin) a peut-être été sentie comme problématique ou, au moins, a pu conduire à préférer cuer .

Dans tous les cas, il ressort de tous ces éléments que, si la distribution, dans la langue vernaculaire, de airme et de cuer s'adapte parfaitement à la distinction entre féminin et masculin mise en place par le latin, il ne faut pas y voir la marque de l'inventivité ou de l'originalité $\mathrm{du}$ traducteur. Que l'équivalence cuer/corage-animus se retrouve dans les autres traductions monastiques est toutefois loin d'être sans intérêt : à l'instar d'autres lexèmes plus rares comme nonsachance, deforainneteiz,

47. G. Merk, « La vitalité des suffixes nominaux du latin au français », Revue de linguistique romane, 34 (1970), p. 194-223 (p. 201).

48. Predigten des $h$. Bernhard in altfranzösischer Übertragung, aus einer Handschrift der königlichen Bibliothek zu Berlin, p. 54 par exemple.

49. Je me permets de renvoyer à mon étude : M.-P. HALARY, La Question de la beauté et le discours romanesque au début du XIII siècle, Paris, 2018, p. 451 sq. 
dedantriens, etc. ${ }^{50}$, l'analyse de ces termes courants semble révéler comme une « communauté lexicale », dont les contours restent à préciser, et on pourrait faire l'hypothèse qu'un discours savant vernaculaire a circulé à l'oral aussi bien qu'à l'écrit, au moins dans les régions du Nord et du Nord-Est. Cette communauté lexicale, selon cette hypothèse, est peut-être latinophone, mais il n'est pas nécessaire qu'elle le soit pour accéder à un discours savant. Ce qui caractérise la langue vernaculaire de ce corpus, c'est qu'elle ne privilégie pas les calques et les latinismes comme le fera ensuite l'interlangue ${ }^{51}$ : bien au contraire, elle accueille un discours hautement spéculatif dans le cadre de la langue commune sans forger un vocabulaire spécifique, à l'instar de ce qui se produit pour le substantif cuer/corage, mot ancien et bien attesté qui actualise ici une acception psychologique voire philosophique.

$\mathrm{Si}$, selon cette proposition, le translateur puise dans un lexique vernaculaire savant partagé quand il doit traduire animus/anima, l'enquête sur la mise en roman de ces termes ne manque pas de révéler une certaine habileté dans la mise en œuvre de ces ressources lexicales. Cela apparaît notamment quand, après la distinction entre l'anima et l'animus, la langue vernaculaire doit rendre compte de la différence entre l'animus et le bonus animus, «l'âme devenue pleinement raisonnable, sortie de la défiguration du péché, ramenée à sa ressemblance avec Dieu, unie à lui par la contemplation et l'amour ${ }^{52}$ » :

Cum enim, liberata voluntate per liberantem gratiam, spiritus agi incipit libera ratione, tunc suus, hoc est seipso libere utens, efficitur, et fit animus et bonus animus ; animus, in quantum bene animans, et perficiens animal suum, supplemento liberae rationis ; bonus autem, in quantum jam amans bonum suum quo bonus fit, et sine quo nec bonus, nec animus, esse potuit. (Epistola..., p. 310)

Et quant li volunteiz est delivreie per la delivrant graice et li esperiz s'encomancet a contenir per franche raison, dons devient siens, c'est dons uset franchement de lui meimes, et si devient cuers et boens cuers. Cuers devient an tant cum il bien ancoraiget et perfait sun airme ${ }^{53}$ per l'acraissement de la delivrance ou il est anfranchis, et boens devient en tant cum il jai aimet son bien per cui il devient boens et sanz cui il ne puet estre ne boens ne cuers. (Li Epistle..., p. 265)

50. Voir G. HASENOHR, « Sur une ancienne traduction lorraine... »; EAD., « Retour sur les caractères linguistiques...».

51. F. Duval, « Le lexique de la civilisation romaine au Moyen Âge : de la diglossie à l'interlinguisme », dans S. Le BrIZ et G. VeYsSEYre éd., Approches du bilinguisme latinfrançais au Moyen Âge. Linguistique, codicologie, esthétique, Turnhout, 2011, p. 63-79.

52. M.-M. Davy, Un traité de la vie solitaire..., p. 147.

53. On peut imaginer une mauvaise lecture ou une leçon avec « animam suam». 
Non seulement, de manière très cohérente, le français reprend le substantif cuer et, à l'image du latin, lui adjoint l'adjectif boen, mais, en outre, il propose un jeu qui, sans reposer sur des latinismes, copie les rapports de dérivation de la langue source : ce que le verbe animare est au substantif animus, le verbe encouragier l'est aux substantifs cuer/corage. Une telle traduction n'avait rien de nécessaire : la seule autre occurrence du verbe latin dans la Lettre est rendue par vivifier ${ }^{54}$. On peut donc comprendre que le souci de précision qui caractérise cette traduction favorise l'exploitation du paradigme de cuer. Plus encore, il favoriserait peut-être même son enrichissement. À la différence du verbe encoragier, dont les dictionnaires montrent qu'il est bien attesté au $\mathrm{XII}^{\mathrm{e}}$ siècle, le nom encoragement est très rare dans la langue médiévale : le Dictionnaire du moyen français indique qu'il n'en existe aucune attestation dans sa documentation et le seul exemple donné par le Dictionnaire de Godefroy (et repris par les autres ouvrages) ${ }^{55}$ est emprunté, précisément, à l'Epistle saint Bernart a Mont Deu. Avec toutes les précautions qu'il convient de prendre avec la notion de néologie ${ }^{56}$, on peut donc penser que le translateur forge un mot nouveau ou, au moins, qu'il recourt là à un substantif assez rare $^{57}$. Son emploi en contexte n'est pas très clair ${ }^{58}$, mais ce qui importe, selon

54. « corpus quod animat » (Epistola..., p. 316) : « lo cors cui il vivifiet » (Li Epistle..., p. 266). Un siècle environ après la composition de cette traduction, Li Ars d'amours, de vertu et de boneurté, autre texte étonnant puisqu'il compile notamment une traduction-adaptation d'Aristote et des translations littérales de la Somme théologique de Thomas d'Aquin, propose également une spéculation en langue vernaculaire sur la nature et les puissances de l'âme. Dans le glossaire (monolingue) qui précède le texte dans deux manuscrits, c'est l'emprunt animans qui est proposé et ainsi défini : " metans arme u vertu d'arme en aucun cors » (Li Ars d'amour, de vertu et de boneurté, éd. J. PetrT, Bruxelles, 1867-1869, t. 1, p. XLVI). Sur ce texte, voir G. Guldentops et C. Steel, « Vernacular philosophy for the nobility: Li Ars d'amour, de vertu et de boneurté, an Old French Adaptation of Thomas Aquinas'Ethics from ca. $1300 »$, Bulletin de philosophie médiévale, 45 (2003), p. 67-85 ; O. COLLET, « Li Ars d'amour, de vertu et de boneurté (c. 1300) : la constitution du lexique philosophique à l'aube d'une nouvelle tradition vernaculaire », dans J. Hamesse et J. Meirinhos éd., Glossaires et lexiques médiévaux inédits. Bilan et perspectives, Porto, 2011, p. 39-55.

55. F. Godefroy, Dictionnaire de l'ancienne langue française..., t. III, p. 116. L'exemple en n. 58 est repris par A. Tobler et E. Lommatzsch, Altfranzösisches Wörterbuch, t. III-1, col. 241, et par le Trésor de la langue française informatisé.

56. F. Duval, « Les néologismes », dans C. Galderisi éd., Translations médiévales..., vol. 1, p. 499-534.

57. C'est ainsi que le doublet synonymique peut être interprété : voir C. BURIDANT, «Les binômes synonymiques. Esquisse d'une histoire des couples de synonymes du Moyen Âge au XVII ${ }^{\mathrm{e}}$ siècle », Bulletin du Centre d'analyse du discours de l'Université de Lille III, Synonymies, 4 (1980), p. 5-76.

58. « $[\ldots]$ et ad exuendam foedae servitutis necessitatem et carnis suae dominantem consuetudinem se accingat » (Epistola...,p. 214) : «por osteir ansus de lui la necessiteit de laide servituit [...] ; oiet ceu et si praignet ancoraigement et air encontre la costume de sa chair ki signerie at sor lui » (Li Epistle..., p. 242). Je comprends mal comment le traducteur en arrive à cette solution. Je suppose que le texte source est un peu différent de celui que donne J. Déchanet et/ou que «prendre ancoraigement et air » explicite le latin se accingere au sens de « se préparer, se disposer à ». 
la perspective qui est développée ici, ce sont les modalités de la formation lexicale. Ces quelques éléments sur le paradigme de cuer (corage, encoragier, encoragement) témoignent en effet de la vitalité de la langue dite vulgaire qui, confrontée au latin savant, relève le défi de la traduction grâce à des mots de construction romane, intelligibles sans la connaissance du latin.

De cette enquête portant sur un détail, il ressort que la version romane de la Lettre d'or accueille un discours savant vernaculaire qui, alors qu'il a parfois toutes les apparences de la langue commune, est extrêmement précis, cohérent et élaboré. Derrière la (fausse) simplicité de cuer et ses dérivés, derrière la polarisation à l'aide des substantifs cuer et airme, c'est bien la complexité du perfectionnement spirituel qui est évoqué : le français devient, sinon une langue de spécialité ${ }^{59}$, au moins une langue de la spéculation religieuse. Or, comme cela a été suggéré, si l'exemple d'une telle vernacularisation reste marginal, il n'est pas complètement isolé : d'autres traductions de textes spirituels procèdent de la même manière et recourent eux aussi aux noms cuer/corage pour parler de l'animus. Outre que de telles translations invitent à nuancer certaines périodisations de l'histoire des traductions et à interroger la distribution sociale des langues (la langue romane n'est-elle que la langue des laïcs ?), elles amènent à reconsidérer le lexique de certains textes et à se demander si, derrière une apparence commune ou banale, il ne possède pas, en réalité, un sens spécialisé.

Dans le prolongement des suggestions faites, en des termes un peu différents, par Geneviève Hasenohr ${ }^{60}$ et Sylvain Piron ${ }^{61}$, il est ainsi permis d'interroger l'origine du vocabulaire de Marguerite Porete. On a souvent remarqué l'influence qu'a sans doute exercée sur le Mirouer la pensée développée dans la Lettre d'or : cette translation ou une traduction de ce type pourrait-elle constituer le « chaînon manquant » permettant de relier Guillaume à Marguerite? La comparaison entre les deux lexiques ${ }^{62}$ pourrait être instructive et, outre la concurrence entre cor et animus pour traduire

59. Sur la question d'un vernaculaire savant, voir les riches contributions proposées dans S. Marcotte et C. Silvi éd., Latinum cedens. Le français et le latin langues de spécialité au Moyen Âge, Paris, 2014.

60. G. HASENOHR, « Retour sur les caractères linguistiques... ».

61. S. PIRON, « Marguerite, entre les béguines et les maîtres », dans S. L. FIELD, R. E. LERNER, S. Piron éd., Marguerite Porete et le Miroir des simples âmes..., p. 69-101 (p. 69-76).

62. Une étude de ce type exige de grandes précautions méthodologiques dans la mesure où, à l'exception du fragment de Valenciennes, le texte primitif du Mirouer est perdu. Il est toutefois possible de faire des conjectures intéressantes à partir des différentes versions qui en dérivent (moyen français, latin et anglais). Sur toutes ces questions, voir surtout l'important article de G. HASENOHR, « La tradition du Miroir des simples âmes au XV siècle : de Marguerite Porète $(† 1310)$ à Marguerite de Navarre », Comptes rendus des séances de l'Académie des inscriptions et belles-lettres, 143/4 (1999), p. 1347-1366. 
corage $^{63}$, il est possible de signaler d'emblée dans les deux textes les jeux sur les paradigmes de franc (anfranchir, franchise) et de encombrer/ descombrer. Si la traduction de Guillaume montre que la pensée monastique peut aussi se dire et s'écrire en langue romane, il n'est peut-être pas aberrant de faire l'hypothèse que le premier grand texte de la mystique française est aussi redevable aux spirituels latins.

\section{Marie-Pascale Halary - Université Lumière-Lyon 2, UMR 5648 (CIHAM)}

\section{Sur une traduction médiévale de la Lettre aux frères du Mont-Dieu. Le témoignage ancien d'un vernaculaire savant}

Cette étude porte sur une traduction ancienne (deuxième moitié du XII siècle), en dialecte lorrain, de la Lettre aux frères du Mont-Dieu de Guillaume de Saint-Thierry. Après avoir rappelé les principales caractéristiques des translations monastiques contemporaines, elle examine les modalités de la mise en roman de ce texte spirituel en se concentrant, notamment, sur la traduction du binôme de substantifs anima/animus. L'enjeu est de montrer l'existence à date ancienne d'une scripturalité vernaculaire savante, qui n'était peut-être pas destinée à un public laïque.

animus/anima, Epistola ad fratres de Monte Dei, Guillaume de Saint-Thierry, littérature spirituelle, traductions monastiques, vernaculaire savant

\section{A medieval translation of William of Saint-Thierry's Letter to the Brothers of Mont-Dieu. Early evidence of a learned vernacular}

This study looks at an early translation (second half of the twelfth century), in the Lorraine dialect, of the Letter to the Brothers of Mont-Dieu by William of Saint-Thierry. After outlining the main characteristics of contemporary monastic translations, it examines how this spiritual text was transposed into this Romance language by focusing in particular on the translation of the pair of nouns " anima/animus. " It aims to demonstrate the existence, at an early date, of a scholarly vernacular scripturality, which may not have been intended for a secular audience.

animus/anima, Epistola ad fratres de Monte Dei, Letter to the Brothers of Mont-Dieu, monastic translations, spiritual literature, vernacular scripturality, William of Saint-Thierry

63. Le texte en moyen français donne au chap. 22 «pour la lascheté de son povre courage » (Marguerite. Porete, Le Mirouer des simples ames, éd. R. Guarnieri, Turnhout, 1986, p. 84), ce qui correspond à l'anglais « for slaknesse of his poore corage » (The Mirror of Simple Souls. A Middle English Translation, éd. M. Dorron, Rome, 1968, p. 275). Or si le manuscrit de base choisi pour l'édition latine contient «propter ignauiam pusillanimis cordis sui » (Margaretae Porete Speculum Simplicium Animarum, éd. P. Verdeyen, Turnhout, 1986, p. 85), les autres donnent « propter ignauiam pauperis animi sui » (ibid.). 